\title{
MULTIDIMENSIONAL MOTION ANALYSIS OF MEMS USING COMPUTER MICROVISION
}

\author{
Dennis M. Freeman \\ freeman@mit.edu
}

\author{
Alexander J. Aranyosi \\ aja@mit.edu
}

\author{
Michael J. Gordon \\ mjgordon@mit.edu
}

\author{
Stanley S. Hong \\ stanhong@mit.edu
}

Department of Electrical Engineering and Computer Science and Research Laboratory of Electronics

Massachusetts Institute of Technology, Cambridge, Massachusetts 02139

\begin{abstract}
Tools for in situ motion analysis of microelectromechanical systems (MEMS) are primitive by comparison to those available for microfabricated electronic systems. A potentially powerful method. which we call computer microvision, results from analysis of video images from a light microscope with algorithms from machine vision. In this paper, we demonstrate two practical applications of computer microvision: to calibrate a MEMS fatigue testing device, and to obtain a modal decomposition of motions of a microfabricated gyroscope.

The fatigue test structure was provided by Failure Analysis Associates, Inc. A proof mass is rotated about its single point of attachment to the substrate under a variety of controlled stimulus and environmental conditions. Motions are induced by electrical stimulation of one comb drive and sensed by the capacitance change in a second comb. Computer microvision was used to measure full six-degree-of-freedom motion of the proof mass. Results provide a direct calibration of the sense signal.

The microfabricated gyroscope is a tuning fork gyroscope provided by The Charles Stark Draper Laboratory. Two proof masses move alternately toward and away from each other in a tuning fork fashion. Angular rotations of the substrate about the axis of symmetry between the proof masses induce out-of-plane motions that are sensed as the output. The gyroscope is structurally complex and supports many modes of motion. Motions of the proof masses were measured using computer microvision, and the results were decomposed into components representing in-plane modes. Measurements were repeated at different frequencies to characterize the frequency dependence of each mode of motion. Modes were well fit as second order resonances. The tuning-fork (difference mode) resonant frequency (in air) was $22.8 \mathrm{kHz}$ with quality of tuning (Q) of 202. The hula (common mode) resonant frequency was $20.9 \mathrm{kHz}$ with a $\mathrm{Q}$ of 162.
\end{abstract}

\section{BACKGROUND}

MEMS are fabricated using VLSI methods similar to those used to fabricate electronic devices. Unlike electronics, however, simple methods for testing and characterizing internal failure modes of micromechanical devices do not exist. This limitation has important consequences for designers and fabricators of MEMS. For the designer, it can result in large numbers of costly prototypes. For the fabricator, it means increased costs because defects go undetected until late in the manufacturing process (e.g.. after packaging). As the demand for precision micromechanical parts increases, and as the complexity of micromechanical systems increases, developing better tools for characterizing the performance of MEMS becomes crucial.
We have investigated tools for in situ measurement of micromechanical motions based on the combination of light microscopy, video imaging, and machine vision. We have previously used such tools to measure cochlear micromechanics [3], and we have also demonstrated the feasibility of their application to MEMS [7]. In this paper, we describe practical applications of the tools to calibrate motions of MEMS, to measure three-dimensional frequency responses of MEMS, and to obtain modal decompositions of motions.

\section{METHODS}

MEMS are viewed with a microscope and imaged with a CCD camera while being driven with a periodic electrical signal (Figure 1). An LED is strobed once per stimulus period at a chosen phase, to yield a snapshot of the device position at the specified phase. This process is repeated at several stimulus phases, and at several focal planes. Computer vision algorithms estimate the change in position of the device between successive images. From these estimates, the periodic motion of the device is determined. This process is repeated at other stimulus frequencies and/or amplitudes to characterize the device motion. Measured motions were decomposed into their predominant modes, and frequency responses of these modes were thereby obtained.

Video microscopy. Test structures were mounted on the stage of a light microscope (Zeiss Axioplan 2 Motorized Research Microscope. Oberkochen, Germany), resting on an active vibration isolation table (Integrated Dynamics Engineering, Woburn MA). The objective (Zeiss LD-Epiplan 20X, numerical aperture 0.4) has a $9.8 \mathrm{~mm}$ working distance, large enough to accommodate the use of external electrical probes. The magnified image was projected onto a scientific grade, 10-bit CCD camera (Kodak Megaplus 1.6i), which has $15.34 \times 1024$ pixels with $9 \mu \mathrm{m}$ spacing.

Stroboscopic illumination. The maximum frame rate of a scientific-grade video imager is typically on the order of tens of Hertz. To image faster motions, we use stroboscopic illumination. A green LED (Nichia NSPG500S, Tokushima 774, Japan) is pulsed so that the target is illuminated at a selected phase of its motion (see waveforms in Figure 1). Light from the LED was collected with a halogen lamp housing: the halogen lamp was removed, and the LED was mounted near the intended position of the tungsten filament. The plastic lens that originally covered the LED's die was milled away and the flat surface was polished, so that illumination was Köhler [11]. Since the intensity of the light emitted from an LED is more nearly proportional to current than voltage, the LED was driven by a computer-controlled D/A converter via a voltageto-current converter. Because LEDs are fast. the shortest light pulse is limited in practice by the electronics driving the LED (roughly $1 \mu$ s for our circuit). 


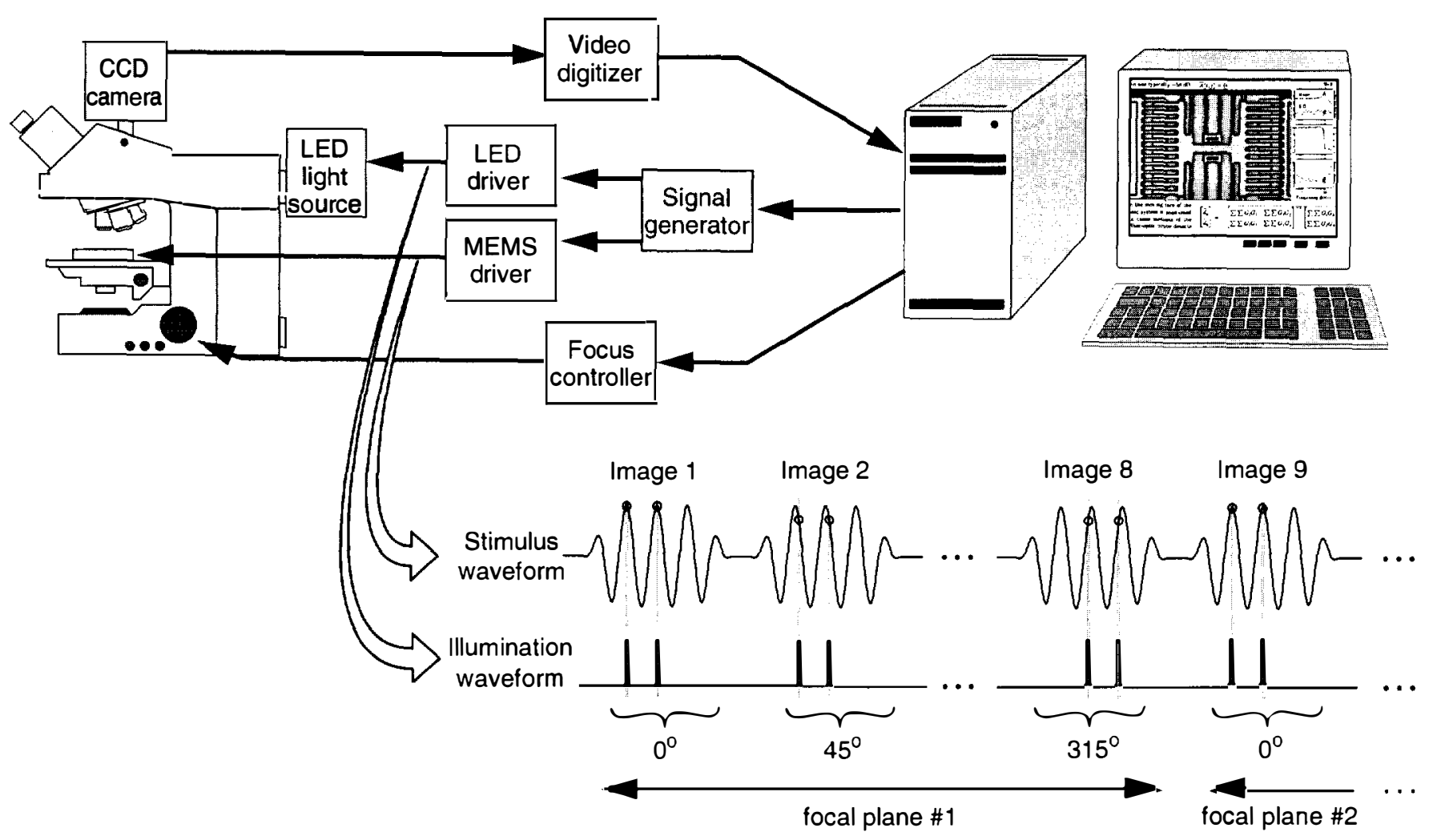

Figure 1. Computer microvision measurement system. The test device is placed on the stage of the microscope (left). A Pentium-based computer controls a signal generator that provides two synchronized waveforms: one to drive motions of the test device and one to strobe the light source. Typical waveforms are shown. Motions are driven with a sinusoidal stimulus. The first image is acquired when light from the LED samples the image at times corresponding to peaks in the stimulus waveform. Successive images are acquired at different phases. This process is then repeated for different focal planes selected by a computer-controlled focus adjustment.

Motion estimates from video images. To characterize sinusoidal motions, eight or sixteen images of the specimen were acquired at evenly-spaced phases of the stimulus cycle using stroboscopic illumination. The LED was gated so that the duty cycle was $1 / 8$ period if eight images were collected or $1 / 16$ period if 16 phases were collected.

Displacements between images acquired at successive phases were estimated directly from the video images. Although image resolution is limited by the optics [8] to distances on the order of the wavelength of light (typically $550 \mathrm{~nm}$ ), displacements between two such images as small as nanometers can be reliably measured [5]. Algorithms for motion detection are described elsewhere $[4 ; 12]$.

Translational and/or rotational displacement estimates at successive phases were used to reconstruct time waveforms of motion. The magnitude and phase of the fundamental component of that waveform were computed with an FFГ. All magnitudes are reported as peak-to-peak values.

3D measurements. Silicon devices are opaque to green light. Nevertheless, information about axial motion can be obtained from images obtained from different focal planes. Images from above the best plane of focus of a structure are blurred. As the structure moves axially, the axial blurring pattern moves with the structure. Therefore, the algorithms that are used to track in-plane motions by computing in-plane gradients can equally track out-of-plane motions by computing out-of-plane gradients [12]. Images were acquired at different focal planes $(50 \mathrm{~nm}$ resolution) to obtain information about
3D motion. In early experiments, focus was adjusted by translating the stage in the axial direction using an integrated stepper motor (part of the Zeiss Axioplan 2 Motorized Research Microscope). This method provided axial positioning with $0.05 \mu \mathrm{m}$ resolution over large axial excursions - essentially limited only by the rack-andpinion mechanism that supported the stage. However, axial motions were accompanied by submicrometer in-plane displacements. In later experiments, focus was controlled by translating the objective with a piezoelectric crystal (PIFOC, Polytec PI, Wandbronn, Germany). Although this device limited axial excursions to $100 \mu \mathrm{m}$, in-plane displacements were significantly smaller.

Modal analysis. MEMS were stimulated sinusoidally, and motions were measured as a function of frequency of the excitation. Frequency responses typically exhibited a small number of resonant peaks that suggest the importance of a small number of resonant modes of motion. When the resonant peaks were sharp, the spatial character of the mode could be determined simply by observing the pattern of motion at the resonant frequency. However, when the resonant peaks were not sharp, empirical methods for separating modes were employed.

One important empirical method takes advantage of common mode and difference mode decompositions of motion measurements at multiple sites. Consider, for example, out-of-plane motion measurements at two edges of a proof mass. The common-mode component of these measurements, which is simply the average of the two axial motions, provides a quantitative estimate of the amount 

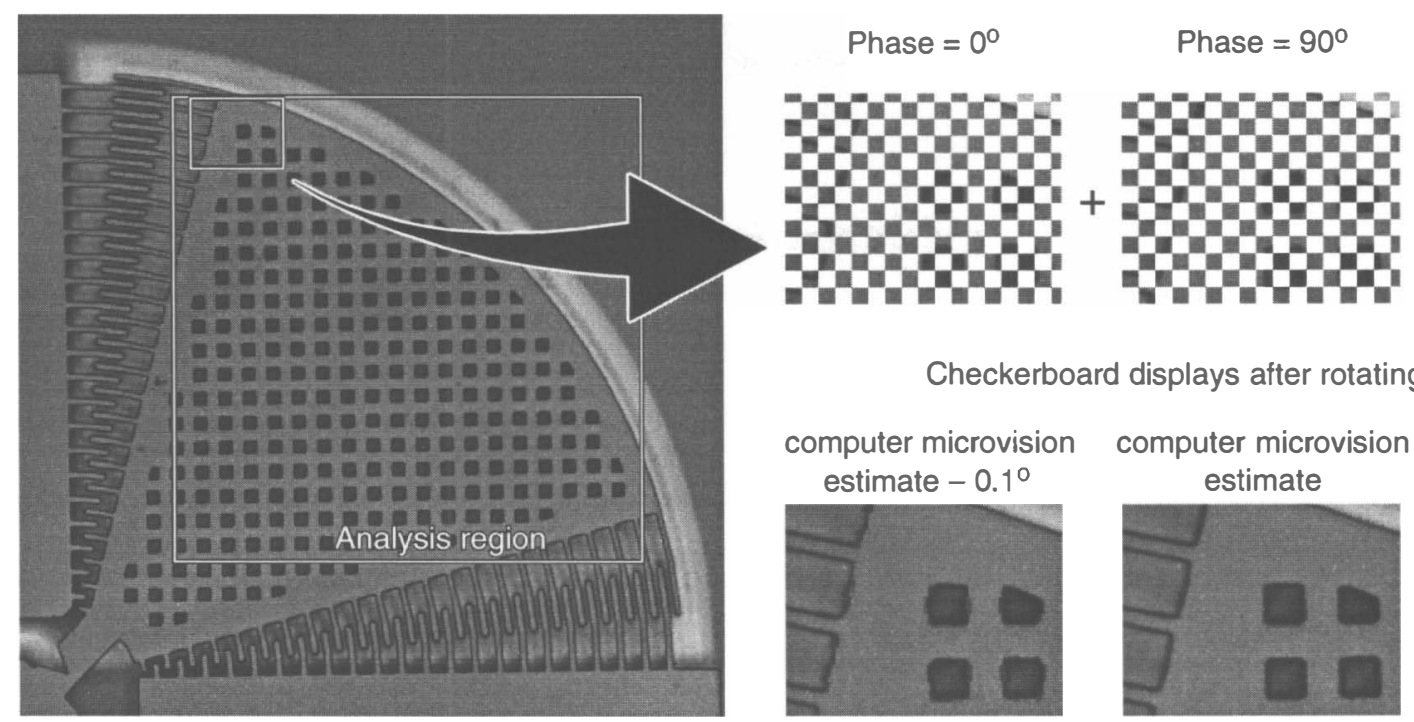

Figure 2. Accuracy of motion estimates using computer microvision. The left panel shows an image of a fatigue test structure designed by Failure Analysis Associates, Inc. One comb drive was stimulated sinusoidally at $20387.9 \mathrm{~Hz}$ to stimulate rotations at the mechanical resonance frequency of $40775.8 \mathrm{~Hz}$. Images were acquired at 8 phases during each period of the electrical stimulus. Pixels in the analysis region indicated by the large white box were analyzed to determine the angular displacement. Portions from the upper right parts of the images taken at two phases were interleaved in a checkerboard fashion as shown in the upper right panel. The lower right panels illustrate similar checkerboard displays after the first image was rotated by the computer microvision estimate and by the estimate plus and minus $0.1^{\circ}$.

of the average levitation of the proof mass. The difference-mode component of these measurements, which is simply the difference of the two axial motions, provides an estimate of out-of-plane rocking. Thus, out-of-plane motions of a single proof mass can easily be decomposed into out-of-plane rocking and out-of-plane levitation modes using the common and difference mode decompositions.

A second important empirical method takes advantage of the fact that modes of motion are invariant to stimulus condition (provided that the stimulus amplitudes are small enough that nonlinearities are not important). Thus modes that are too small to measure under one stimulus condition can sometimes be measured using a different stimulus condition. Consider out-of-plane levitation as an example. Little out-of-plane levitation would be expected if two comb drives located on opposite sides of a proof mass provide pushpull excitations. Thus, motions in response to this stimulus condition would not provide much information about the resonant frequency or quality of tuning of the levitation mode. In this case, symmetrical electrical stimulation is useful to characterize out-of-plane levitation even if the normal stimulus condition is push-pull.

\section{RESULTS}

We have applied computer microvision to calibrate the motions of fatigue test structures, to measure in-plane and out-of-plane frequency responses of those structures, and to obtain modal decompositions of motions of microfabricated tuning fork gyroscopes.

\section{Calibration of fatigue test structures from Failure Analy-} sis Associates, Inc.

Failure Analysis Associates, Inc. has developed test structures to investigate effects of stimulus and environmental conditions, as well as manufacturing process conditions, on the performance, aging, and ultimate failure of MEMS [2]. The test structures consist of a proof mass that is rotated about its single point of attachment to the substrate (Figure 2, left panel). Motions are induced by electrical excitation of one comb drive and are sensed by the change in capacitance of the other comb. The goal is to control environmental conditions, to monitor changes in responses, and to formulate quantitative models of reliability for MEMS.

An important response property for development of quantitative reliability models is the angular displacement of the proof mass. However, direct measurements of angular displacement are difficult. By comparison, indirect measurements of angular displacement that are based on the change in capacitance of an integrated comb are relatively simple. These indirect measurements are especially important for long duration tests of a large number of devices. However, the same process variations and/or aging processes that can affect the mechanical properties of the fatigue test structure could, at least in principle, affect the relation between angular displacement and capacitance between the combs. Consider for example that out-ofplane bending of only a small fraction of a degree could significantly change the capacitance between the outermost teeth. To directly control for this possibility, we have applied computer microvision to calibrate motions of test structures developed by Failure Analysis Associates, Inc.

Test structures were excited with a variety of electrical stimuli and images were acquired and analyzed by computer microvision. Because the resulting motion estimates are derived directly from sequences of images, the quantitative results can be checked qualitatively by simply viewing the slow-motion video sequences. For example, to test that the rotation estimate between two images is qualitatively correct, the first image can be rotated by the estimate and then compared to the second. To the extent that the estimate is correct, the rotated first image should exactly align with the origi- 
nal second image. Such a qualitative analysis is shown in Figure 2, where registration errors are emphasized in a checkerboard display. Jaggedness of the edges of structures in the checkerboard display indicates registration error between the images.

As shown in Figure 2, visual assessment of alignment can convincingly detect registration errors on the order of $0.1^{\circ}$. This is in good agreement with fundamental limitations of optics [8]. Visual assessments are diffraction limited, i.e., limited by blurring intrinsic to the optics, which is on the order of the wavelength of light for well-corrected optics. Blurring of images from our system, which uses green light (wavelength $=550 \mathrm{~nm}$ ), is on the order of $0.5 \mu \mathrm{m}$. Thus, errors in alignment on the order of the blurring width translate to angular errors on the order of $\arctan (0.5 / L)$, where $L$ is the length of the structure in $\mu \mathrm{m}$. For the test structure in Figure 2 $L \approx 400 \mu \mathrm{m}$, suggesting blurring errors on the order of $0.07^{\circ}$.

Although images are blurred by diffraction, they can be accurately registered to within a small fraction of the blurring radius using algorithms from machine vision [9]. We use a gradient method [10] that relates changes in brightnesses across images to gradients in brightness within an image. For example, if a pixel is illuminated by a middle part of a target that is brighter to the right and dimmer to the left, then the pixel should get brighter if the target moves to the left and dimmer if the target moves to the right. The gradient algorithm combines changes in brightnesses of all pixels in a region of interest (large white box in left panel of Figure 2) using a least squares method $[4 ; \mathbf{5}]$. Figure 3 show results of this algorithm for 5 independent repetitions of the measurement at 15 different amplitudes of stimulation. The standard deviation for repeated trials is less than 4 milli-degrees p-p at all amplitudes.

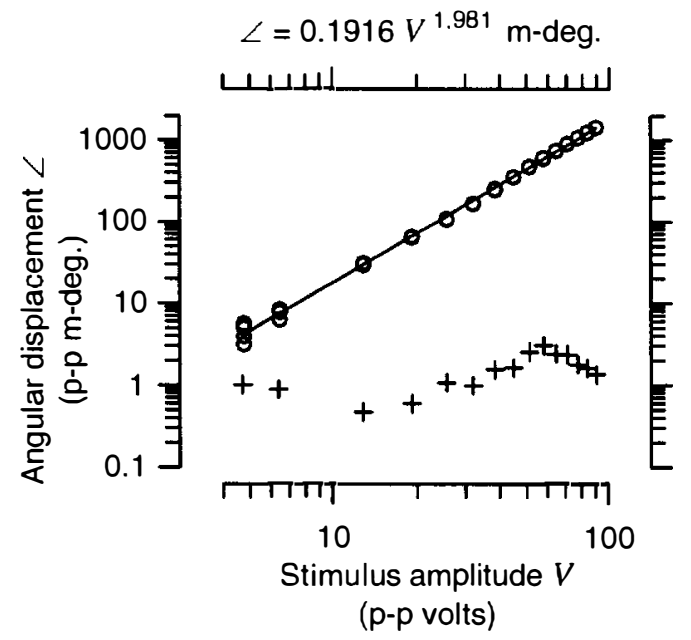

Figure 3. Calibration of fatigue test structure developed by Failure Analysis Associates, Inc. The device used in Figure 2 was stimulated at resonance with a sinusoidal voltage. Five independent measurements (circles) were made at each of 15 different amplitudes (abscissa) of stimulation. The line given by the equation at the top of the plot is a regression line fit by a least squares method. Pluses indicate the standard deviations of results at each amplitude.

Analysis of images at multiple planes of focus provides threedimensional motion information (see Methods). Three-dimensional measurements at multiple points on the proof mass of the fatigue test structure allow determination of three-dimensional angular displacements. Figure 4 illustrates angular displacements as a function of stimulus frequency. These measurements exhibit high quality res- onance for angular displacements in the plane of the proof mass and low-pass behaviors for out-of-plane levitation and rocking.
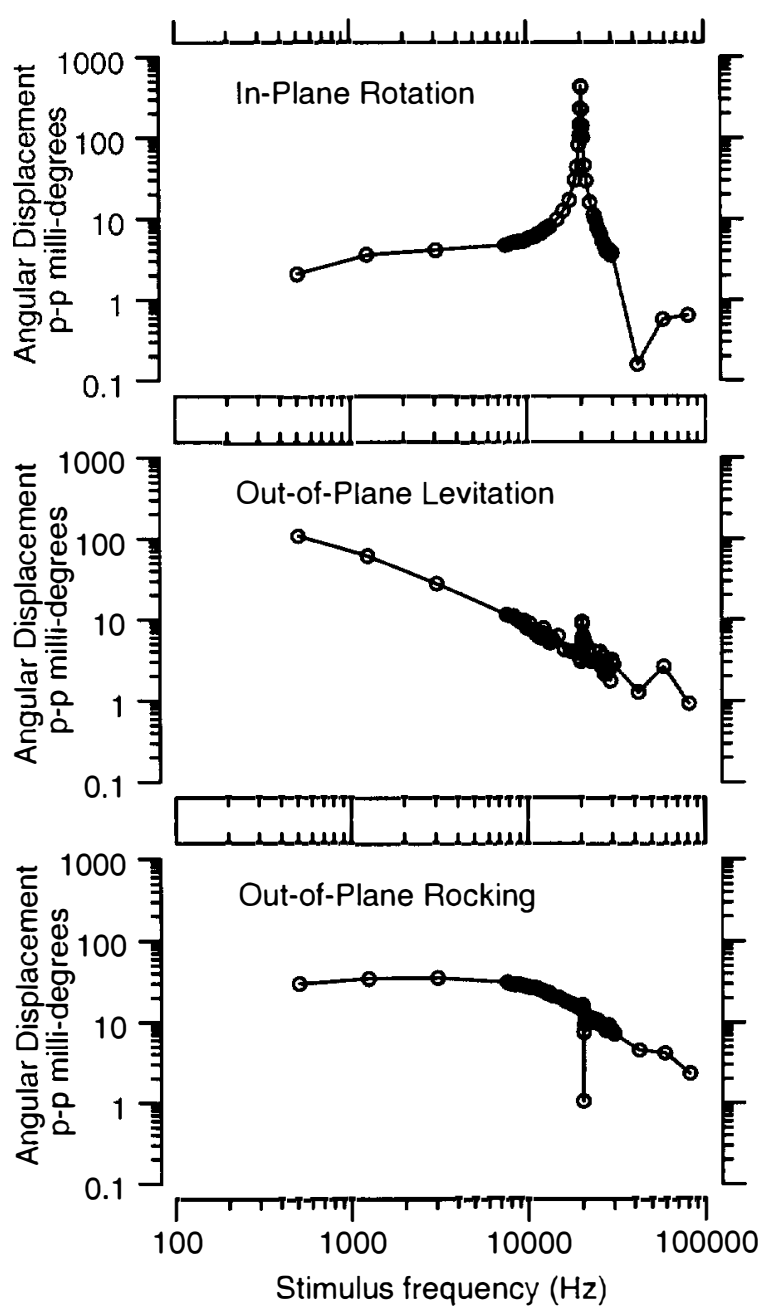

Figure 4. Three-dimensional rotations of fatigue test structure. Motions were excited with constant amplitude $(60 \mathrm{~V} p$-p) stimuli at 56 frequencies. Mechanical responses are at twice the stimulus frequency. Images at 12 focal planes (2 $\mathrm{\mu m}$ spacing) were analyzed to determine in-plane rotation, out-of-plane levitation, and out-of-plane rocking. Results are displayed as angular displacements. The outlier point in the out-of-plane rocking measurements falls exactly on the in-plane resonance frequency.

\section{Modal analysis of the Draper gyroscope}

The Charles Stark Draper Laboratory has developed a microfabricated gyroscope for sensing angular velocity [1]. The gyroscope consists of two proof masses suspended by cantilevers. The masses are driven by comb drives along one axis, so that angular rotation about the perpendicular in-plane axis induces a coriolis force in the out-of-plane direction. This force induces motion of the proof masses perpendicular to the substrate, and the motion is sensed as a change of capacitance between the proof mass and the substrate. The two masses are driven in opposite directions to facilitate separation of angular velocity from linear acceleration.

The gyroscope is structurally complex and supports many 
modes of motion; the dominant modes of motion in the plane of the proof masses are a tuning-fork mode (the difference mode of the two proof masses), and a hula mode (the common mode of the two proof masses). The hula mode can complicate the sensing of angular velocity by adding unwanted sensitivity to linear acceleration. Draper Labs has applied design and simulation tools to analyze undesired modes of motion. We have extended these efforts by experimentally measuring displacements of the proof masses and determining the contribution of in-plane modes of motion to these displacements.

Motions of the proof masses were measured using computer microvision under three stimulus conditions. In stimulus condition A, the outer combs of both proof masses were driven with a $40 \mathrm{~V} p-p$ sinusoid with $20 \mathrm{~V}$ DC offset; this stimulus preferentially excites the tuning-fork mode. In stimulus condition $\mathrm{B}$, the outer comb of the left-hand proof mass was driven; this stimulus preferentially excites the hula mode. In stimulus condition $\mathrm{C}$, both combs of the lefthand proof mass were driven with the same voltage. This stimulus preferentially excites the out-of-plane levitation mode.

In all stimulus conditions, both tuning-fork and hula modes were induced. To estimate tuning properties of the tuning-fork mode, we computed half the difference in motion of the two proof masses in stimulus condition A. To estimate tuning properties of the hula mode, we computed half the sum of the motions of the two proof masses in stimulus condition $\mathrm{B}$. The magnitude of the frequency response of each mode was fit with a low-pass second-order resonance. Each fit had three free parameters - DC gain, resonant frequency, and quality of tuning $(\mathrm{Q})$. The measured and fitted phases were compared later to determine the goodness of the fit. Both modes were well fit by second-order resonances. The tuning-fork (difference mode) resonant frequency (in air) was $22.8 \mathrm{kHz}$ with a Q of 202. The hula (common mode) resonant frequency was $20.9 \mathrm{kHz}$ with a $\mathrm{Q}$ of 162 . Figure 5 shows the measured modes and computed fits as a function of frequency.

Motions of any point on a structure can be described as a weighted sum of the dominant modes of motion of the structure [6]. For example, in-plane motions of one proof mass of the gyroscope can be fit with a weighted sum of the tuning-fork and hula modes. The weights are the DC gains of the modes, which vary with stimulus condition and level and determine the contribution of each mode to the overall motion. The sum of the tuning-fork and hula modes gives a four-pole, two-zero system where the relative weights of the modes determine the locations of the zeroes, but not of the poles. The magnitude of motion of one proof mass was fit by this four-pole, two-zero system with two free parameters: one (gain) affecting the overall level of the fit, and one (relative mode weights) affecting the shape by changing the frequency of the zeroes. The fit was performed for the left proof mass under stimulus condition $\mathrm{A}$, and for the right proof mass under stimulus condition C. As with the modal fits, a post hoc comparison of phase was done to determine the goodness of the fit. Motion of the proof masses under both stimulus conditions was well fit by the weighted sum of the modes. In stimulus condition A, the tuning-fork mode had a DC gain of $0.0525 \mu \mathrm{m}$, and the hula mode had a DC gain of 0.00277 $\mu \mathrm{m}$. In stimulus condition $\mathrm{C}$, the tuning-fork mode had a DC gain of $0.00604 \mu \mathrm{m}$, and the hula mode had a DC gain of $0.00101 \mu \mathrm{m}$. Figure 6 shows the measured displacements and computed fits as a function of frequency.

\section{DISCUSSION}

We have demonstrated the use of computer microvision as a tool for in situ motion analysis of MEMS. We have employed this
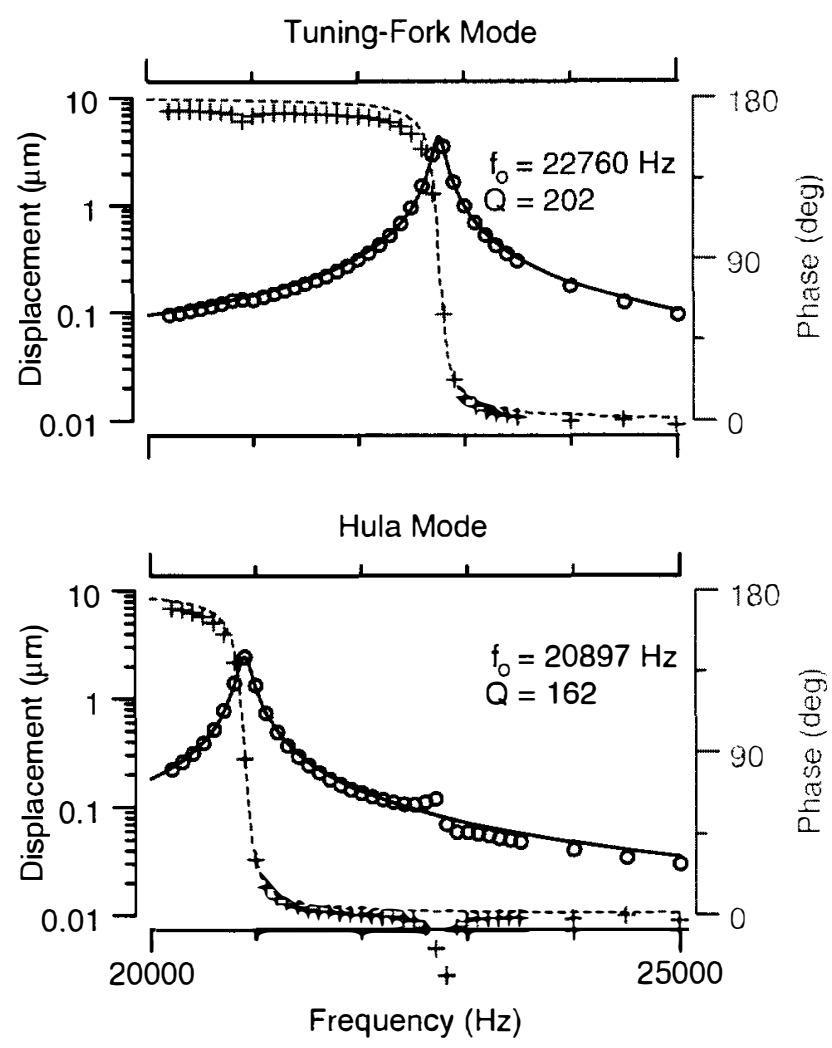

Figure 5. Modes of motion of the Draper gyroscope. Proof masses were driven by $40 \mathrm{~V}$ peak-to-peak sinusoid with $20 \mathrm{~V} \mathrm{DC}$ offset. Displacements of both proof masses in the direction of excitation were measured at each frequency using computer microvision. These displacements were subtracted and divided by 2 to estimate the tuning-fork component, and were summed and halved to estimate the hula component. The modes were then fit with low-pass second-order resonances. The plots show the measured (symbols) and fitted (lines) magnitude (black circles) and phase (gray pluses) of the two modes. A. Tuning-fork mode. This mode was preferentially excited by driving the outer combs of both proof masses (stimulus condition A). Resonant frequency $=22760 \mathrm{~Hz}$; damping coefficient $=0.00247$; mean squared error $=1.13 \mathrm{~nm}$. B. Hula mode. This mode was preferentially excited by driving the outer comb of the left proof mass (stimulus condition B). Resonant frequency $=20897 \mathrm{~Hz}$; damping coefficient $=0.03085$; mean squared error $=0.660 \mathrm{~nm}$.

new tool to calibrate motions of a fatigue test structure and to perform a modal decomposition of motions of a microfabricated gyroscope.

These results demonstrate several unique features of computer microvision. First, computer microvision provides estimates of translations and rotations in multiple dimensions. Measurements in multiple dimensions allow unambiguous characterization of complex motions. This contrasts with other measurement methods, such as laser interferometry or capacitive sensing, which provide an estimate of just one component of motion. Second, motion estimates with computer microvision are derived directly from sequences of images. Thus, all quantitative results can be checked qualitatively by simply viewing those images (Figure 2 ). This contrasts with other measurement systems, such as laser interferometry or capacitive sensing, which provide no internal consistency checks. Third, computer microvision provides concurrent measurements of all im- 

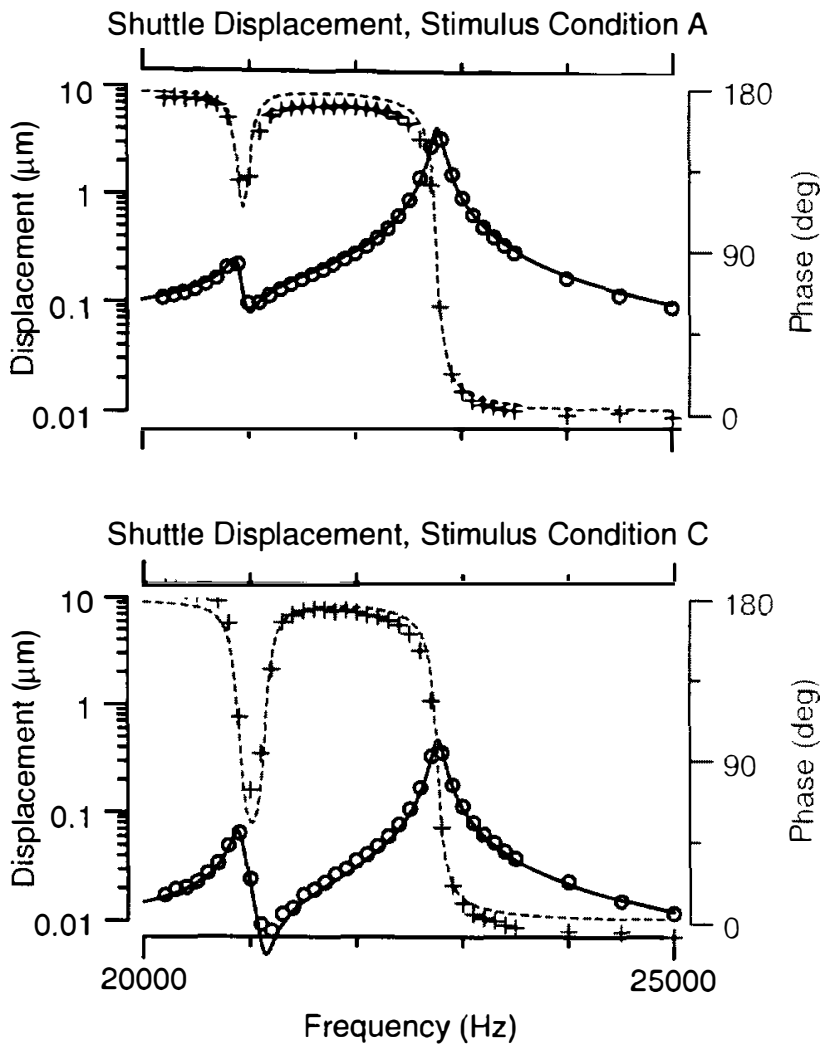

Figure 6. Modal reconstruction of proof mass displacement. Both plots show the displacement of a proof mass as a function of frequency. These displacements were fit with a weighted sum of the two modes shown in Figure 5. Symbols are as in Figure 5. A. Displacement of the left proof mass, outer combs of both masses driven (stimulus condition $A$ ). The hula mode weight was 0.0528 relative to the tuning-fork mode. Mean squared error $=1.84 \mathrm{~nm}$. B. Displace ment of the right proof mass, both combs of left proof mass driven (stimulus condition $C$ ). The hula mode weight was 0.1673 relative to the tuning-fork mode. Mean squared error $=0.243 \mathrm{~nm}$.

aged structures. Motion measurements for different structures can be important for modal analysis (Figure 5). This contrasts with other measurement systems, such as laser interferometry or capacitive sensing, which measure motions of just one part of a structure.

Results in this paper demonstrate how computer microvision can be used to quantify motions of any imaged structure, and to relate motions of multiple structures to characterize complex modes of interaction. Modal analyses based on calibrated motion measurements provide important new opportunities for understanding the mechanical properties of MEMS.

\section{ACKNOWLEDGMENTS}

The authors acknowledge contributions to this project by previous colleages, including C. Quentin Davis, who developed the first computer microvision system, Zoher Z. Karu, who developed the three-dimensional algorithms, Laura K. Johnson, who first applied the method to analyze motions of the Draper gyroscope, and William W. Van Arsdell, who first applied the method to analyze motions of the fatigue test structure.
The authors gratefully acknowledge support of Failure Analysis Associates, Inc. and The Charles Stark Draper Laboratory. We especially thank Chris Muhlstein and Stuart Brown at Failure Analysis Associates, Inc. and Marc Weinberg, Jim Campbell, and John Bernstein for supplying test devices and for helping with measurements and interpretations. This work was supported by grants from DARPA (F30602-97-2-0106) and from The Charles Stark Draper Laboratory (E-96-414). This work builds on research in hearing supported by the NIH (2 R01 DC00238), which includes support for A. J. Aranyosi and D. M. Freeman. D. M. Freeman was supported in part by the W. M. Keck Career Development professorship.

\section{REFERENCES}

1. J. Bernstein, S. Cho, A. T. King, A. Kourepenis, P. Maciel, and $\mathbf{M}$. Weinberg. A micromachined comb-drive tuning fork rate gyroscope. In Solid-State Sensor and Actuator Workshop, pages 143-148. Transducer Research Foundation, Inc., Cleveland, June 1993.

2. S. B. Brown, W. Van Arsdell, and C. L. Muhlstein. Materials reliability in MEMS devices. In Transducers '97, pages 591593. 1997 International conference on Solid-State Sensors and Actuators, Chicago, June 1997.

3. C. Q. Davis and D. M. Freeman. Direct observations of sound-induced motions of the reticular lamina, tectorial membrane, hair bundles, and individual stereocilia. In Abstracts of the Eighteenth Midwinter Research Meeting. St. Petersburg Beach, Florida, February 1995. Association for Research in Otolaryngology.

4. C. Q. Davis and D. M. Freeman. Statistics of subpixel registration algorithms based on spatio-temporal gradients or block matching. Optical Engineering, 1998. (to appear in April).

5. C. Q. Davis and D. M. Freeman. Using a light microscope to measure motions with nanometer accuracy. Optical Engineering, 1998. (to appear in April).

6. D. G. Fertis. Mechanical and Structural Vibrations. John Wiley and Sons, Inc., New York, NY, 1995.

7. D. M. Freeman and C. Q. Davis. Using video microscopy to characterize micromechanics of biological and manmade micromachines. In Solid-State Sensor and Actuator Workshop, pages 161-167. Transducer Research Foundation, Inc., June 1996.

8. H. H. Hopkins and P. M. Barham. The influence of the condenser on microscopic resolution. Proc. Phys. Soc., 63:737-744, 1950.

9. B. K. P. Horn. Robot Vision. MIT Press, Cambridge, MA, 1986.

10. B. K. P. Horn and E.J. Weldon. Jr. Direct methods for recovering motion. Intematl. J. of Computer Vision, 2:51-76, 1988.

11. Shinya Inoué. Video Microscopy. Plenum Press, New York, NY, 1986.

12. Z. Z. Karu. Fast subpixel registration of 3-D images. PhD thesis, Massachusetts Institute of Technology, 1997. 